\title{
ANDES

\section{Comportamiento alimentario, suficiencia dietaria y estado nutricional en niños entre 6 a 18 meses}

\author{
Feeding behavior, dietary sufficiency and nutritional status in children between 6 and \\ 18 months
}

\author{
Edson Bustos A. ${ }^{\oplus a}$, Marcelo Cádiz C. ${ }^{\circledR a}$, Karina Etchegaray A. ${ }^{\bullet a}$, Oscar Castillo V. ${ }^{\oplus a}$
}

aNutricionista. Escuela de Nutrición y Dietética, Facultad de Medicina, Universidad Finis Terrae. Santiago, Chile

Recibido: 26 de noviembre de 2020; Aceptado: 17 de marzo de 2021

¿Qué se sabe del tema que trata este estudio?

Existen escasos estudios que aborden este tema en el grupo de niños entre 6 a 18 meses. La literatura se concentra en la Lactancia Materna (LM), y no especialmente en el inicio y continuación de la Alimentación Complementaria (AC).
¿Qué aporta este estudio a lo ya conocido?

Entrega información adicional tanto de LM como AC para identificar aspectos propios del comportamiento alimentario y suficiencia dietaria de este grupo de niños. Además aporta conocimiento actualizado sobre el estado nutricional en niños con diferente Nivel Socioeconómico (NSE).

\section{Resumen}

La alimentación infantil presenta características cualitativas y cuantitativas específicas. La influencia de los padres sobre la ingesta de alimentos es crucial en el desarrollo de hábitos y un adecuado estado nutricional. Objetivo: Evaluar el comportamiento alimentario, las características dietarias y estado nutricional en niños entre 6 a 18 meses. Sujetos y Método: Estudio descriptivo de corte transversal en 199 niños. En una muestra por conveniencia se consignaron datos demográficos y antropométricos para estado nutricional, y antecedentes dietarios, mediante recordatorio de $24 \mathrm{~h}$ para el comportamiento dietario y la suficiencia alimentaria. Se aplicó t-student y ANOVA de una vía para variables continuas y test chi-cuadrado para categóricas. Resultados: 54,8\% mujeres. Estado nutricional: $21,1 \%$ sobrepeso y $7,6 \%$ obesidad. Un $56,8 \%$ de los niños tenían números de comidas acorde su edad. La ingesta promedio de energía fue de $652,9 \pm 224,2$ calorías diarias. La ingesta de energía y macronutrientes fue significativamente mayor en los niños que no consumían leche materna y de 12 a 18 meses. La adecuación energética, lípidos y carbohidratos en las niñas fue significativamente mayor a la de niños. Un $83,1 \%$ del consumo de alimentos fueron habituales para este grupo etario. Conclusión: En el comportamiento alimentario se encontró que 4 de cada 10 niños presentan números de comidas mayor al sugerido a su edad. En cuanto a la suficiencia dietaría hay una mayor ingesta en los niños con estado nutricional normal, sin LM y entre 12 y 18 meses. A pesar de la alta prevalencia de obesidad, esta fue menor a la observada a nivel nacional.

Correspondencia:

Edson Bustos A.

edsonbustos@uft.cl 


\begin{abstract}
Infant feeding has specific qualitative and quantitative characteristics. The influence of parents on food intake is crucial in the development of habits and an adequate nutritional state. Objective: To evaluate eating behavior, dietary characteristics, and nutritional status of children between 6 to 18 months. Subjects and Method: Cross-sectional descriptive study in 199 children. Using convenience sampling, demographic and anthropometric data were recorded for nutritional status, and dietary history, through a 24-hour reminder for dietary behavior and food sufficiency. The Student's t-test and one-way ANOVA were applied for continuous variables and the chi-square test for the categorical ones. Results: $54.8 \%$ were women. Regarding nutritional status, $21.1 \%$ were overweight and $7.6 \%$ obese. $56.8 \%$ of the children had meal numbers according to their age. The average energy intake was $652.9 \pm 224.2$ calories per day. The intake of energy and macronutrients was significantly higher in children not consuming breast milk and aged 12 to 18 months. The energy, lipids, and carbohydrates adequacy in girls was significantly higher than in boys. $83.1 \%$ of food consumption was habitual for this age group. Conclusion: In eating behavior, we observed that 4 out of 10 children have meal numbers higher than suggested for their ages. Regarding dietary sufficiency, there is a higher intake in children with normal nutritional status, without breastfeeding, and between 12 and 18 months. Despite the high prevalence of obesity, it was lower than that observed at the national level.
\end{abstract}

\section{Keywords:}

Nutritional Status;

Foods;

Nutrients;

Energy;

Feeding Behavior;

Children

\section{Introducción}

Los hábitos alimentarios, ingesta dietética y estado nutricional de los lactantes y niños durante los primeros 2 años de vida son de gran relevancia. Durante esta etapa la lactancia materna, y el inicio y consolidación de la alimentación complementaria están destinadas a cubrir los altos requerimientos nutricionales para la mantención de un adecuado estado nutricional y de salud. Además, ambos tipos de alimentación son instancias primordiales en la educación alimentaria entregada por los padres, así como en la generación de hábitos y preferencias alimentarias, los cuales en gran medida se replicarán y mantendrán durante la niñez y edades futuras ${ }^{1,2}$.

En el lactante, la alimentación complementaria inadecuada, la malnutrición materna y la ausencia o parcialidad de la lactancia materna pueden tener repercusiones negativas, directas o indirectas, sobre la salud del niño, el crecimiento pondoestatural, riesgo de patologías agudas e infecciosas, obesidad o desnutrición y de enfermedades autoinmunes y atópica ${ }^{3}$.

En el 2003, la Organización Mundial de la Salud (OMS) y el Fondo de las Naciones Unidas para la Infancia (UNICEF) publicaron un documento en el que recalcaban que las prácticas de alimentación inadecuadas son un factor de riesgo importante de morbimortalidad en la primera infancia ${ }^{4}$. En la Guía de Alimentación del Niño(a) Menor de 2 años publicada en el 2015 por el Ministerio de Salud de Chile (MINSAL) 5 , se señala la relevancia de la alimentación en los primeros 1.000 días de vida ${ }^{6}$, los vínculos afectivos y el apego del niño con la madre, así como las recomendaciones en alimentación normal, hábitos y conducta alimentaria para niños sanos ${ }^{2}$. En el 2017 en el documento sobre alimentación complementaria de la Sociedad Europea de Gastroenterología, Hepatología y Nutrición (ESPGHAN), se hace énfasis en las diferencias culturales en las prácticas alimentarias entre los países, incentivando la lactancia materna, promoviendo la inclusión segura, paulatina y de buena calidad nutricional de diversos alimentos en la alimentación complementaria (incluidos los potencialmente alergénicos) y alentando a los padres a responder a las señales de hambre y saciedad, sin sobrealimentar ni entregar alimentos como premio o recompensa ${ }^{7}$.

Respecto al estado nutricional de los niños chilenos en el año 2016 el MINSAL reportó que en el rango etario de 7-11 meses y en el de 12-17 meses, la prevalencia de obesidad y sobrepeso osciló entre 10,1\% a 10,2\% y entre $24,8 \%$ a $25,6 \%$ respectivamente. Estas cifras son alarmantes, situando a Chile como uno de los países con la más alta prevalencia de sobrepeso y obesidad a nivel mundial ${ }^{8}$.

Sin embargo, existen muy escasos estudios internacionales que evidencien sobre el comportamiento alimentario en lactantes o que determinen la ingesta de nutrientes detallada durante este periodo. Los estudios encontrados muestran una alimentación poco variada, alta en lácteos y carnes, pero bajas en legumbres, pescados y alimentos ricos en ácidos grasos esenciales ${ }^{9}$. Entre los estudios más relevantes se encuentran cuatro estudios de cohorte: el "Estudio de cohorte de madre e hijo de Noruega" $(\mathrm{MoBa})^{10}$, el "Estudio de diseño longitudinal antropométrico y nutricional de Dortmund" (DONALD $)^{11}$, el "Estudio longitudinal de embarazo e infancia de Avon" (ALSPAC) ${ }^{12}$ y el estudio de estratos de población susceptible "Impacto en la salud pública 
de la exposición a elementos mixtos de bajo nivel a largo plazo" (PHIME) ${ }^{13}$. Es importante destacar que no se han encontrado estudios a nivel nacional que indaguen sobre el comportamiento alimentario y la ingesta en este grupo etario.

El objetivo del estudio es evaluar el comportamiento alimentario, las características dietarias, hábitos y estado nutricional en niños entre 6 a 18 meses en la ciudad de Santiago de Chile.

\section{Sujetos y Método}

Se realizó un estudio descriptivo de corte transversal durante los meses de junio a agosto 2017 en niños entre 6 a 18 meses de la ciudad de Santiago de Chile. La selección de la muestra fue por conveniencia y se consideró como criterio de inclusión niños nacidos de término, de peso adecuado para la edad gestacional y con inicio regular al sexto mes de vida de la alimentación complementaria. Además, se hizo una estratificación del nivel socioeconómico (NSE) familiar basada en la categoría ocupacional y el nivel educacional de ESOMAR $^{14}$. Estás categorías fueron reagrupadas en tres grupos quedando como NSE alto: ABC1, NSE medio: C2, C3 y NSE bajo: $\mathrm{D}^{15}$.

Las madres y/o cuidadores de 256 niños fueron contactados por vía telefónica a través de la agencia de estudios públicos Gfk Adimark y posteriormente visitadas en sus domicilios por un equipo de nutricionistas entrenadas en antropometría y registros dietarios. En la primera visita se informó a todos los sujetos del protocolo de estudio y solicitó la aceptación y firma del consentimiento informado, finalmente aceptaron participar e incluyeron 199 niños para este estudio. En la segunda visita las nutricionistas registraron los antecedentes sociodemográficos y evaluaron antropométricamente a los niños, se midió el peso corporal en kilos y gramos con una balanza digital marca SECA modelo 354 (Hamburgo, Alemania), con una graduación de $10 \mathrm{~g}$ y capacidad máxima de 20 kilos, la longitud a través del infantómetro móvil marca SECA modelo 417 (Hamburgo, Alemania), con graduación de 1 milímetro y un rango de medición de 10-100 centímetros. Además, se capacitó a las madres a realizar el autorregistro digital en línea de la encuesta de recordatorio de $24 \mathrm{~h}$ de cuatro a siete días, incluyendo al menos un día de fin de semana, se educó para registrar horario, tipo y cantidad de alimento ingerido por el niño y su equivalencia en gramos o mililitros. También se les entregó a las madres el contacto telefónico de las nutricionistas en el caso de preguntas en la aplicación de la encuesta recordatorio $24 \mathrm{~h}$. Los datos extraídos de los registros digitales de las encuestas de recordatorio de $24 \mathrm{~h}$. fueron analizados con el Software Food Processor II ver- sión 11.3, utilizando aquellos alimentos que sean idénticos o tengan mayor similitud en sus características nutricionales a los alimentos chilenos. Este software ha sido validado y utilizado previamente por nuestro grupo $^{16}$. En el caso de la leche materna solo se registró su ingesta de manera cualitativa por la imposibilidad de cuantificarla.

La codificación y manejo de los datos se llevaron a cabo por nutricionistas especializados en el Software Food Processor II y se consideraron para el análisis nutricional: energía (Kcal), proteínas totales $(\mathrm{g})$, lípidos (g), omega $3(\mathrm{~g})$, omega 6 (g), carbohidratos (g), vitaminas: vitamina A como equivalente de retinol $(\mu \mathrm{g})$, vitamina $\mathrm{D}(\mu \mathrm{g})$, vitamina $\mathrm{E}$ como $\alpha$-tocoferol equivalente $(\mathrm{mg})$, vitamina $\mathrm{K}(\mu \mathrm{g})$, vitamina $\mathrm{C}(\mathrm{mg})$, tiamina $(\mathrm{mg})$, riboflavina $(\mathrm{mg})$, niacina $(\mathrm{mg})$, piridoxina $(\mathrm{mg})$, folato $(\mu \mathrm{g})$, vitamina B12 $(\mu \mathrm{g})$, ácido pantoténico $(\mathrm{mg})$ y biotina $(\mu \mathrm{g})$ y minerales: calcio $(\mathrm{mg})$, fósforo $(\mathrm{mg})$, cobre $(\mu \mathrm{g})$, flúor $(\mathrm{mg})$, yodo $(\mu \mathrm{g})$, hierro $(\mathrm{mg})$, zinc $(\mathrm{mg})$, potasio $(\mathrm{g})$ y sodio $(\mathrm{g})$.

Los antecedentes antropométricos fueron tratados bajo los estándares de la Organización Mundial de la Salud (OMS) 2006 $6^{17,18}$. Se definió "malnutrición por déficit" (MD) como puntaje $\mathrm{zP} / \mathrm{E} \leq-1$ en menores de 12 meses y $\mathrm{zP} / \mathrm{T} \leq-1$ para mayores de 12 meses (riesgo de desnutrir: $\mathrm{zP} / \mathrm{E}$ o $\mathrm{zP} / \mathrm{T}-1$ a $-1,99$; desnutrición: $\mathrm{zP} / \mathrm{E}$ o zP/T $\leq-2$, según edad que corresponda). Se definió "malnutrición por exceso" (ME) como puntaje $\mathrm{zP} / \mathrm{T} \geq+1$ (sobrepeso: $\mathrm{zP} / \mathrm{T}+1 \mathrm{a}+1,99$; obesidad $\mathrm{zP} / \mathrm{T}$ $\geq+2$ ). Además, se utilizó como referencia de hábitos y conductas alimentarias saludables las contenidas en la Guía de Alimentación del Niño(a) Menor de 2 años, publicada por el Ministerio de Salud (MINSAL) de Chile el año $2015^{5}$. Finalmente, se consideró el consumo de alimentos procesados y ultra procesados, descritos como aquellas formulaciones principalmente de fuentes industriales baratos en energía y alimentos dietéticos más aditivos que utilizan una serie de proce$\operatorname{sos}^{19}$, suelen tener un alto contenido de grasas totales, grasas saturadas, azúcar y sal añadida, junto con una menor densidad de fibra y vitaminas ${ }^{20}$.

\section{Consideraciones éticas}

Este estudio respetó las exigencias de la declaración de Helsinki de la Asociación Médica Mundial sobre ética en la investigación médica en seres humanos y la normativa actualmente vigente en nuestro país y fue aprobado por el Comité de Bioética de la Universidad Finis Terrae. Se solicitó el consentimiento informado a los padres o cuidadores legales de los niños incluidos en nuestro estudio.

\section{Análisis estadístico}

Se verificó la normalidad de las variables con test de Shapiro Wilk. Las variables numéricas se describie- 
ron como promedios y desviación estándar. Para cada niño, la ingesta promedio diaria de energía, macronutrientes y micronutrientes se calcularon sobre la base de los registros de 4 a 7 días de Recordatorios de $24 \mathrm{~h}$, excluyendo el uso de suplementos. La ingesta de energía fue comparada con la estimación de requerimientos energéticos según los Principios y aplicación de las nuevas necesidades de energía según el Comité de Expertos FAO/OMS $2004^{21}$, mientras que la ingesta proteica y micronutrientes fue comparada con la Dietary Reference Intakes $2006^{22}$, se consideró una adecuación normal de la ingesta alimentaria real respecto a los requerimientos nutricionales de cada niño, cuando para energía y macronutrientes esta adecuación estaba entre el $90-110 \%$ y para vitaminas y minerales entre un 70 $130 \%{ }^{22}$.

Aquellas variables de dos categorías fueron comparadas con $t$ de Student y las variables que presentaron tres o más niveles fueron comparadas con la prueba de ANOVA de una vía y test de comparaciones múltiples de Bonferroni. Las variables categóricas se describieron según frecuencias. Se consideró significativo un $\mathrm{p}<0,05$ y se utilizaron los programas estadísticos SAS 9.4 y SPSS 17.

\section{Resultados}

En la tabla 1 puede observarse que ingresaron al estudio 199 niños (edad 12,2 $\pm 3,9$ meses), 54,8\% de sexo femenino, $51,7 \%$ de nivel socioeconómico medio, 28,7\% presentó ME (sobrepeso + obesidad), un $53,8 \%$ consumía leche materna y un $43,2 \%$ consumió un mayor número de comidas diarias a las recomendadas.

La tabla 2 muestra la ingesta energética, macronutrientes y micronutrientes críticos de todos los niños según su consumo de leche materna y rango de edad. Se observaron diferencias significativas en el mayor consumo de energía, macronutrientes, minerales y vitaminas $\mathrm{A}, \mathrm{D}, \mathrm{E}$ y $\mathrm{C}$, excepto en vitamina $\mathrm{K}$, en los niños entre 12 a 18 meses y quienes no consumían leche materna en su dieta diaria. La ingesta de energía de la muestra total fue de 652,9 \pm 224,2 calorías. No se encontraron diferencias significativas en la ingesta energética según sexo, edad y estado nutricional. En el análisis de macronutrientes y micronutrientes críticos (vitaminas A, E, D, K, C y minerales como calcio, hierro y Zinc) tampoco hubo diferencias significativas en la ingesta por sexo y estado nutricional. Al evaluar el porcentaje de adecuación según NSE (ABC1 vs. D) en energía (86,0 $\pm 28,0$ vs. $92,4 \pm 33,7)$ y proteínas $(178,5 \pm 71,3$ vs. $198,1 \pm 94,7)$, encontramos una adecuación normal para energía y elevada para proteínas. En cuanto, al porcentaje de adecuación de lípidos, carbohidratos y micronutrientes críticos no se encontraron diferencia significativa según NSE.

En la tabla 3, se muestran los porcentajes de adecuación de la muestra completa y comparada según sexo. Cabe destacar que aun cuando la media de adecuación para energía de toda la muestra estuvo dentro de la normalidad, la adecuación de lípidos, omega 3 y 6 , vitaminas $A, D, E$, niacina, biotina y minerales como cobre flúor, yodo, potasio y sodio estuvieron por debajo de lo esperado. Mientras que proteínas, carbohidratos, vitaminas $\mathrm{K}, \mathrm{C}$ y $\mathrm{B} 12$ tuvieron una media por sobre lo establecido como adecuado. Al comparar según sexo, se observó que para energía, lípidos y carbohidratos las niñas presentaron una adecuación significativamente mayor en comparación con los niños.

En la tabla 4 se compararon los porcentajes de adecuaciones según estado nutricional, se observó que los niños con MD y normal tuvieron una media de adecuación de energía dentro del rango normal, no así los con ME estuvo levemente por debajo. Además, tuvimos que la media de adecuación para lípidos, omega 3 y 6 , vitaminas A, D, E, niacina, biotina y minerales como flúor, yodo y sodio estuvieron por debajo de lo esperado en los tres grupos de estado nutricional, siendo importante señalar que el sodio en el grupo de MD tuvo una adecuación significativamente mayor comparado al grupo de ME, aun cuando para ambos grupos estaba por debajo de lo normal. En el caso del potasio, se observó que solo era adecuada en el grupo de MD y fue significativamente mayor al compararlo con el grupo normal y ME. En cambio, la media de adecuación para proteínas, carbohidratos, vitaminas $\mathrm{K}, \mathrm{C}$ y B12 estuvieron por sobre lo establecido como adecuado en los tres grupos, y al comparar específicamente los carbohidratos, se observó que los niños con MD tuvieron una adecuación significativamente mayor a los niños con ME, aun cuando en los tres grupos nutricionales estaba elevada.

La totalidad de alimentos consumidos se agruparon en 13 categorías las que fueron evaluadas según sexo, rango de edad y estado nutricional (tabla 5). Se observó que el $83,1 \%$ de los alimentos consumidos fueron los habituales para la edad, especialmente leche materna, cereales, frutas, verduras, leches, carnes/legumbres y aceites crudos. El 12,9\% consumía alimentos procesados (ej: colados infantiles) y además un 3,9\% de consumo de alimentos ultra procesados como embutidos, azúcares y snacks salados, estas observaciones se dieron para sexo, rango de edad y estado nutricional. Mientras que la adición de edulcorantes artificiales se observó en mujeres con estado nutricional normal y en ambos grupos etarios. 


\section{Tabla 1. Características generales de la muestra}

\begin{tabular}{|c|c|c|}
\hline Características & $\mathrm{n}$ & $\%$ \\
\hline $\begin{array}{l}\text { Sexo } \\
\text { Masculino } \\
\text { Femenino }\end{array}$ & $\begin{array}{r}90 \\
109\end{array}$ & $\begin{array}{l}45,2 \\
54,8\end{array}$ \\
\hline $\begin{array}{l}\text { Rango de edad } \\
6-11 \text { meses } \\
12-18 \text { meses }\end{array}$ & $\begin{array}{r}93 \\
106\end{array}$ & $\begin{array}{l}46,7 \\
53,3\end{array}$ \\
\hline $\begin{array}{l}\text { Nivel socioeconómico (NSE) } \\
\text { ABC1 } \\
\text { C2 } \\
\text { C3 } \\
\text { D }\end{array}$ & $\begin{array}{l}33 \\
54 \\
49 \\
63\end{array}$ & $\begin{array}{l}16,6 \\
27,1 \\
24,6 \\
31,7\end{array}$ \\
\hline $\begin{array}{l}\text { Estado nutricional } \\
\text { Desnutrición } \\
\text { Riesgo de desnutrir } \\
\text { Normal } \\
\text { Sobrepeso } \\
\text { Obesidad }\end{array}$ & $\begin{array}{r}1 \\
17 \\
124 \\
42 \\
15\end{array}$ & $\begin{array}{r}0,5 \\
8,5 \\
62,3 \\
21,1 \\
7,6\end{array}$ \\
\hline $\begin{array}{l}\text { Diagnóstico de talla } \\
\text { Baja } \\
\text { Normal } \\
\text { Alta }\end{array}$ & $\begin{array}{r}8 \\
178 \\
13\end{array}$ & $\begin{array}{r}4,0 \\
89,5 \\
6,5\end{array}$ \\
\hline $\begin{array}{l}\text { Ingesta de leche materna } \\
6-11 \text { meses } \\
12-18 \text { meses }\end{array}$ & $\begin{array}{r}107 \\
66 \\
41\end{array}$ & $\begin{array}{l}53,8 \\
70,9 \\
38,7\end{array}$ \\
\hline $\begin{array}{l}\text { Número de comidas diarias según recomendación (total) } \\
6 \text { - } 11 \text { meses ( } 5 \text { a } 6 \text { comidas diarias) } \\
12-18 \text { meses ( } 4 \text { a } 5 \text { comidas diarias) }\end{array}$ & $\begin{array}{r}113 \\
78 \\
35\end{array}$ & $\begin{array}{l}56,8 \\
83,9 \\
33,0\end{array}$ \\
\hline Edad en meses (promedio y DS) & 12,2 & 3,9 \\
\hline
\end{tabular}

Tabla 2. Ingesta de calorías, macronutrientes y micronutrientes críticos según consumo de leche materna (LM) y rango de edad

\begin{tabular}{|c|c|c|c|c|c|c|c|}
\hline \multirow[t]{2}{*}{ Nutrientes } & Todos los niños & $\begin{array}{l}\text { Con LM } \\
(n=107)\end{array}$ & $\begin{array}{l}\operatorname{Sin} L M \\
(n=92)\end{array}$ & $\mathrm{p}$ & $\begin{array}{c}6 \text { a } 11 \text { meses } \\
(n=93)\end{array}$ & $\begin{array}{c}12 \text { a } 18 \text { meses } \\
(n=106)\end{array}$ & \multirow[t]{2}{*}{$p$} \\
\hline & Media $\pm \mathrm{DE}$ & Media $\pm \mathrm{DE}$ & Media $\pm \mathrm{DE}$ & & Media $\pm \mathrm{DE}$ & Media $\pm \mathrm{DE}$ & \\
\hline Energía (Kcal) & $652,9 \pm 224,2$ & $522,9 \pm 199,2$ & $803,3 \pm 147,7$ & 0,00 & $546,9 \pm 228,2$ & $746,6 \pm 176,9$ & 0,00 \\
\hline Proteínas (g) & $23,2 \pm 10,1$ & $18,7 \pm 9,8$ & $28,6 \pm 7,5$ & 0,00 & $17,9 \pm 8,3$ & $28,0 \pm 9,2$ & 0,00 \\
\hline Lípidos (g) & $21,9 \pm 9,0$ & $16,2 \pm 6,9$ & $28,6 \pm 6,1$ & 0,00 & $18,1 \pm 8,8$ & $25,3 \pm 7,7$ & 0,00 \\
\hline Carbohidratos (g) & $128,8 \pm 53,6$ & $112,4 \pm 53,5$ & $148,1 \pm 47,0$ & 0,00 & $118,3 \pm 60,0$ & $138,2 \pm 129,4$ & 0,00 \\
\hline Vit. A, ER $(\mu \mathrm{g})$ & $227,5 \pm 161,0$ & $157,6 \pm 120,6$ & $309,6 \pm 164,2$ & 0,00 & $180,7 \pm 152,6$ & $269,7 \pm 157,3$ & 0,00 \\
\hline Vit. $D(\mu \mathrm{g})$ & $1,6 \pm 1,5$ & $0,9 \pm 1,2$ & $2,5 \pm 1,4$ & 0,00 & $1,3 \pm 1,8$ & $1,9 \pm 1,2$ & 0,01 \\
\hline Vit. E, $\alpha-T E(m g)$ & $2,5 \pm 2,0$ & $1,8 \pm 1,4$ & $3,2 \pm 2,3$ & 0,00 & $1,7 \pm 1,5$ & $3,1 \pm 2,1$ & 0,00 \\
\hline Vit. K ( $\mu g)$ & $27,2 \pm 31,9$ & $27,7 \pm 31,1$ & $26,6 \pm 32,9$ & 0,81 & $25,8 \pm 34,0$ & $28,4 \pm 30,0$ & 0,56 \\
\hline Vit. C (mg) & $39,8 \pm 31,1$ & $28,1 \pm 21,9$ & $53,5 \pm 34,6$ & 0,00 & $29,0 \pm 26,0$ & $49,5 \pm 32,2$ & 0,00 \\
\hline Calcio (mg) & $442,5 \pm 289,9$ & $275,6 \pm 209,7$ & $638,6 \pm 245,2$ & 0,00 & $325,2 \pm 276,5$ & $548,5 \pm 260,3$ & 0,00 \\
\hline Hierro (mg) & $9,4 \pm 3,9$ & $7,1 \pm 3,1$ & $12,0 \pm 3,1$ & 0,00 & $8,2 \pm 4,2$ & $10,4 \pm 3,4$ & 0,00 \\
\hline Zinc (mg) & $3,2 \pm 1,9$ & $2,1 \pm 1,3$ & $4,5 \pm 1,6$ & 0,00 & $2,4 \pm 1,7$ & $4,0 \pm 1,7$ & 0,00 \\
\hline
\end{tabular}

ER: equivalente de retinol; $\alpha$-TE: $\alpha$-tocoferol equivalente. T-test $p<0,05$. 
Tabla 3. Porcentaje de adecuación de ingesta dietaria por sexo

\begin{tabular}{|c|c|c|c|c|}
\hline Nutrientes & $\begin{array}{c}\text { Todos los niños } \\
\text { Media } \pm \text { DE }\end{array}$ & $\begin{array}{c}\text { Niñas } \\
\text { Media } \pm \text { DE }\end{array}$ & $\begin{array}{c}\text { Niños } \\
\text { Media } \pm \text { DE }\end{array}$ & $p$ \\
\hline Energía & $89,7 \pm 30,8$ & $94,8 \pm 29,2$ & $83,5 \pm 31,7$ & 0,01 \\
\hline Proteínas & $189,9 \pm 90,3$ & $197,5 \pm 82,8$ & $180,6 \pm 10,3$ & 0,19 \\
\hline Lípidos & $64,2 \pm 26,5$ & $67,6 \pm 25,3$ & $60,2 \pm 27,4$ & 0,04 \\
\hline Omega 3 & $3,4 \pm 8,1$ & $2,3 \pm 2,9$ & $4,6 \pm 11,5$ & 0,07 \\
\hline Omega 6 & $47,9 \pm 40,7$ & $47,0 \pm 37,8$ & $48,9 \pm 43,9$ & 0,75 \\
\hline Carbohidratos & $141,5 \pm 62,7$ & $150,0 \pm 60,1$ & $131,2 \pm 64,7$ & 0,03 \\
\hline Vit. A, ER & $66,7 \pm 51,1$ & $69,4 \pm 55,9$ & $63,5 \pm 44,8$ & 0,41 \\
\hline Vit. D & $13,0 \pm 12,4$ & $13,4 \pm 11,9$ & $12,5 \pm 13,1$ & 0,61 \\
\hline Vit. E, $\alpha$-TE & $46,4 \pm 35,4$ & $45,3 \pm 30,9$ & $47,7 \pm 40,2$ & 0,64 \\
\hline Vit. K & $707,4 \pm 1383,9$ & $685,8 \pm 1167,6$ & $732,9 \pm 1608,8$ & 0,81 \\
\hline Vit. C & $196,4 \pm 201,1$ & $216,9 \pm 219,0$ & $172,0 \pm 175,7$ & 0,11 \\
\hline Tiamina & $74,4 \pm 50,0$ & $77,1 \pm 49,4$ & $71,1 \pm 50,8$ & 0,39 \\
\hline Riboflavina & $115,9 \pm 83,5$ & $124,2 \pm 78,6$ & $106,0 \pm 88,4$ & 0,13 \\
\hline Niacina & $39,9 \pm 32,3$ & $39,6 \pm 31,8$ & $40,2 \pm 33,0$ & 0,90 \\
\hline Piridoxina & $97,1 \pm 54,7$ & $97,4 \pm 48,8$ & $96,6 \pm 61,3$ & 0,92 \\
\hline Folato & $80,9 \pm 59,3$ & $81,6 \pm 55,2$ & $80,2 \pm 64,1$ & 0,86 \\
\hline Vit. B12 & $166,6 \pm 170,4$ & $182,7 \pm 168,3$ & $147,4 \pm 171,7$ & 0,14 \\
\hline Ac. Pantoténico & $73,2 \pm 48,5$ & $76,6 \pm 47,6$ & $69,1 \pm 49,6$ & 0,28 \\
\hline Biotina & $64,4 \pm 55,8$ & $61,5 \pm 51,4$ & $67,8 \pm 60,6$ & 0,43 \\
\hline Calcio & $103,9 \pm 85,4$ & $110,2 \pm 76,8$ & $96,5 \pm 94,7$ & 0,27 \\
\hline Fósforo & $100,2 \pm 69,1$ & $107,5 \pm 62,4$ & $91,3 \pm 75,9$ & 0,10 \\
\hline Cobre & $65,2 \pm 35,5$ & $64,9 \pm 90,9$ & $65,6 \pm 40,4$ & 0,89 \\
\hline Flúor & $2,1 \pm 5,4$ & $1,8 \pm 1,5$ & $2,4 \pm 7,7$ & 0,47 \\
\hline Yodo & $19,2 \pm 21,0$ & $18,4 \pm 19,8$ & $20,2 \pm 22,4$ & 0,56 \\
\hline Hierro & $113,1 \pm 56,5$ & $118,3 \pm 58,1$ & $106,7 \pm 54,2$ & 0,14 \\
\hline Zinc & $106,9 \pm 62,3$ & $112,1 \pm 60,1$ & $100,7 \pm 64,8$ & 0,20 \\
\hline Potasio & $62,0 \pm 47,3$ & $64,7 \pm 48,6$ & $58,6 \pm 45,3$ & 0,36 \\
\hline Sodio & $47,7 \pm 31,3$ & $49,9 \pm 20,0$ & $45,1 \pm 33,8$ & 0,29 \\
\hline
\end{tabular}

ER: equivalente de retinol; $\alpha$-TE: $\alpha$-tocoferol equivalente. T-test $p<0,05$.

\section{Discusión}

En este estudio, respecto a comportamiento alimentario se observó en la muestra que un $43,2 \%$ del total de los niños, $16,1 \%$ de los niños entre 6-11 meses y $67 \%$ de los niños de 12-18 meses de edad, presentaba un consumo de número de comidas diarias mayor al sugerido por la Guía de Alimentación del Niño(a) Menor de 2 años, Minsal 20155, la cual recomienda entre 5 a 6 tiempos de comidas para niños entre 6 a 11 meses y 4 a 5 comidas diarias para niños entre 12-18 meses. Se ha descrito en población infantil que el aumento del número de comidas, incluyendo mayor cantidad de colaciones o "picoteos", independiente de la ingesta energética y de macronutrientes diaria, podría ser un factor de riesgo para la aparición de obesidad ${ }^{23}$, así como la alteración de las señales de hambre y saciedad en niños que están adquiriendo hábitos alimentarios ${ }^{24}$. Esta costumbre de picotear entre comidas alcanza a un $47 \%$ de los padres chilenos de niños entre 0 a 12 años ${ }^{25}$, y es conocida la influencia de los hábitos alimentarios de los padres sobre los niños ${ }^{26}$.

Se observó igualmente que un $83,1 \%$ de los alimentos consumidos fueron de las categorías de los alimentos habituales que se incorporan a esta edad, y además se encontró que un $12,9 \%$ del consumo fue de colados 
Tabla 4. Comparación de porcentaje de adecuación de ingesta dietaria versus requerimientos nutricionales, por estado nutricional

\begin{tabular}{|c|c|c|c|c|}
\hline Nutrientes & $\begin{array}{c}\text { Malnutrición por déficit } \\
\text { Media } \pm \mathrm{DE}\end{array}$ & $\begin{array}{c}\text { Normal } \\
\text { Media } \pm \text { DE }\end{array}$ & $\begin{array}{c}\text { Malnutrición por exceso } \\
\text { Media } \pm \text { DE }\end{array}$ & $p$ \\
\hline Energía & $97,5 \pm 31,9$ & $90,4 \pm 30,6$ & $85,5 \pm 30,9$ & 0,32 \\
\hline Proteínas & $215,3 \pm 132,8$ & $191,5 \pm 84,2$ & $178,3 \pm 86,8$ & 0,30 \\
\hline Lípidos & $68,0 \pm 25,5$ & $64,4 \pm 25,9$ & $62,7 \pm 28,4$ & 0,75 \\
\hline Omega 3 & $1,9 \pm 1,6$ & $3,8 \pm 9,6$ & $3,0 \pm 5,5$ & 0,59 \\
\hline Omega 6 & $45,3 \pm 31,6$ & $46,7 \pm 43,1$ & $51,2 \pm 38,3$ & 0,76 \\
\hline Carbohidratos & $174,5 \pm 59,2^{a}$ & $141,2 \pm 66,4$ & $131,6 \pm 52,3^{a}$ & 0,04 \\
\hline Vit. A, ER & $59,7 \pm 37,1$ & $66,8 \pm 55,7$ & $68,8 \pm 44,3$ & 0,80 \\
\hline Vit. D & $13,4 \pm 12,1$ & $13,7 \pm 13,0$ & $11,4 \pm 11,2$ & 0,53 \\
\hline Vit. $E, \alpha$-TE & $48,0 \pm 34,0$ & $46,1 \pm 36,8$ & $46,6 \pm 33,3$ & 0,97 \\
\hline Vit. K & $1.217,6 \pm 1627,8$ & $612,5 \pm 1.383,6$ & $746,7 \pm 1.285,8$ & 0,21 \\
\hline Vit. C & $140,9 \pm 153,9$ & $209,0 \pm 217,6$ & $186,5 \pm 174,2$ & 0,37 \\
\hline Tiamina & $72,1 \pm 44,5$ & $74,2 \pm 52,7$ & $75,5 \pm 46,4$ & 0,96 \\
\hline Riboflavina & $111,6 \pm 82,3$ & $118,6 \pm 78,7$ & $111,4 \pm 94,7$ & 0,84 \\
\hline Niacina & $43,7 \pm 31,4$ & $39,8 \pm 33,6$ & $39,0 \pm 30,2$ & 0,86 \\
\hline Piridoxina & $101,8 \pm 58,2$ & $98,6 \pm 58,8$ & $92,1 \pm 43,6$ & 0,71 \\
\hline Folato & $95,0 \pm 68,7$ & $78,7 \pm 55,6$ & $81,5 \pm 64,5$ & 0,54 \\
\hline Vit. B12 & $171,9 \pm 158,5$ & $177,1 \pm 165,4$ & $141,8 \pm 184,6$ & 0,43 \\
\hline Ac. Pantoténico & $66,9 \pm 44,7$ & $77,6 \pm 51,0$ & $65,5 \pm 43,6$ & 0,25 \\
\hline Biotina & $61,5 \pm 29,5$ & $67,8 \pm 65,1$ & $57,8 \pm 36,7$ & 0,53 \\
\hline Calcio & $136,8 \pm 95,3$ & $102,4 \pm 74,7$ & $97,0 \pm 102,0$ & 0,21 \\
\hline Fósforo & $111,3 \pm 70,1$ & $103,0 \pm 63,6$ & $90,7 \pm 79,8$ & 0,42 \\
\hline Cobre & $77,4 \pm 34,2$ & $62,2 \pm 36,8$ & $67,8 \pm 32,4$ & 0,19 \\
\hline Flúor & $2,2 \pm 1,7$ & $2,4 \pm 6,8$ & $1,5 \pm 1,2$ & 0,59 \\
\hline Yodo & $14,7 \pm 16,7$ & $21,4 \pm 22,9$ & $16,0 \pm 17,2$ & 0,19 \\
\hline Hierro & $112,4 \pm 43,9$ & $111,1 \pm 55,6$ & $117,5 \pm 62,6$ & 0,77 \\
\hline Zinc & $112,0 \pm 62,5$ & $106,8 \pm 61,4$ & $105,5 \pm 65,4$ & 0,92 \\
\hline Potasio & $91,0 \pm 54,9^{a, b}$ & $60,6 \pm 49,4^{b}$ & $55,8 \pm 36,5^{a}$ & 0,01 \\
\hline Sodio & $65,0 \pm 36,8^{a}$ & $46,9 \pm 29,9$ & $44,1 \pm 31,4^{a}$ & 0,04 \\
\hline
\end{tabular}

ER: equivalente de retinol; $\alpha$-TE: $\alpha$-tocoferol equivalente. Prueba de ANOVA de una vía y test de comparaciones múltiples de Bonferroni. $a, b p<0,05$

o picados procesados, altamente disponibles en el comercio. Mostrando que se cumple con las recomendaciones chilenas que fomentan el inicio de la alimentación complementaria a partir del sexto mes de vida, la cual debe ser paulatina y conlleva a diversificar la ingesta de alimentos, cuyos alimentos habituales incluidos a partir de este periodo son lácteos (leche materna o fórmulas lácteas infantiles), carnes, pescados, legumbres, huevo, cereales, papas, arroz, fideos, otros cereales, frutas, verduras y aceites crudos ${ }^{5}$. Lo cual muestra que las recomendaciones son altamente seguidas por los padres y cuidadores en la alimentación de los niños. También encontramos un consumo preocupante de embutidos, azúcares y snacks salados en ambos sexos, rangos etarios y estado nutricional, así como el consumo de edulcorantes artificiales añadidos en mujeres de ambos rangos de edad y con estado nutricional normal. Los cuales, a pesar de no ser porcentajes elevados, no forman parte de ninguna recomendación alimentaria para niños de esta edad, sexo o clasificación nutricional $^{5}$, pero que son altamente adquiridos y consumidos a nivel de la población chilena ${ }^{27,28}$. Cabe destacar, que el consumo de edulcorantes artificiales podría ser mayor, ya que solo se consideró en los registros la adición voluntaria de estos, y no los alimentos que traen añadido edulcorantes dentro de sus ingredientes. Estos 
Tabla 5. Frecuencia de consumo de los diferentes grupos de alimentos consumidos según sexo, edad y estado nutricional (\%)

\begin{tabular}{|c|c|c|c|c|c|c|c|}
\hline \multirow[t]{2}{*}{ Grupos de alimentos } & \multicolumn{2}{|c|}{ Sexo } & \multicolumn{2}{|c|}{ Rango de edad } & \multicolumn{3}{|c|}{ Estado nutricional } \\
\hline & Niña & Niños & 6 a 11 meses & 12 a 18 meses & $\begin{array}{l}\text { Malnutrición } \\
\text { por déficit }\end{array}$ & Normal & $\begin{array}{c}\text { Malnutrición } \\
\text { por exceso }\end{array}$ \\
\hline Leche materna & 9,2 & 10,0 & 16,4 & 4,9 & 8,6 & 9,8 & 9,4 \\
\hline Cereales* & 18,0 & 15,9 & 17,5 & 16,8 & 20,1 & 17,9 & 14,1 \\
\hline Frutas & 11,9 & 12,6 & 11,3 & 12,8 & 10,4 & 11,9 & 13,6 \\
\hline Verduras & 12,9 & 11,5 & 9,8 & 13,9 & 12,4 & 11,5 & 13,9 \\
\hline Carnes" & 2,4 & 2,3 & 0,8 & 3,4 & 1,3 & 2,3 & 2,9 \\
\hline Legumbres & 1,9 & 1,8 & 1,2 & 2,3 & 2,0 & 1,7 & 2,1 \\
\hline Leches $^{\&}$ & 21,9 & 22,8 & 20,7 & 23,4 & 22,0 & 22,2 & 22,5 \\
\hline Colados $^{\circ}$ & 11,8 & 14,0 & 16,3 & 10,4 & 13,6 & 12,8 & 12,5 \\
\hline Aceites & 5,8 & 5,4 & 3,7 & 7,0 & 5,7 & 5,4 & 6,2 \\
\hline Embutidos & 0,2 & 0,3 & 0,1 & 0,3 & 0,6 & 0,2 & 0,2 \\
\hline Azúcares $^{\infty}$ & 3,7 & 3,2 & 2,1 & 4,4 & 3,1 & 4,0 & 2,4 \\
\hline Snack salados $\$$ & 0,1 & 0,2 & 0,1 & 0,2 & 0,2 & 0,1 & 0,2 \\
\hline Edulcorantes artificiales & 0,2 & 0,0 & 0,0 & 0,2 & 0 & 0,2 & 0 \\
\hline Total & $100 \%$ & $100 \%$ & $100 \%$ & $100 \%$ & $100 \%$ & $100 \%$ & $100 \%$ \\
\hline
\end{tabular}

*Cereales: cereales infantiles, papas, arroz, fideos y otros cereales. "Carnes: carnes rojas, carnes blancas, pescados y huevo. Leches: leches infantiles, fórmulas lácteas y otros lácteos. ${ }^{\circ} \mathrm{Colados}$ c colados y picados infantiles comerciales. ${ }^{\infty} \mathrm{Azúcares:} \mathrm{azúcar} \mathrm{añadida} \mathrm{y} \mathrm{snacks} \mathrm{altos} \mathrm{en}$ azúcar. ${ }^{\$}$ Snack salados: snack salados y alimentos altos sodio y grasas.

alimentos han aumentado en Chile posterior a la aplicación de la Ley de Alimentos 20.606 "Sobre Composición Nutricional de los Alimentos y su Publicidad"29, ya que con ello la industria busca disminuir el número de sellos de alto en calorías y azúcares en sus alimentos, pero se desconoce si su ingesta habitual y elevada puede tener efectos adversos, más aún en niños ${ }^{30}$.

En suficiencia dietaria no se encontraron diferencias significativas en la ingesta energética, macronutrientes y micronutrientes críticos según sexo y estado nutricional, aun así, la ingesta promedio energética en niños con MD y normales fue mayor que en niños con ME, lo mismo se observó en su adecuación energética. Esto concuerda con lo encontrado en otros estudios donde se observó mayor ingesta en niños con estado nutricional normal ${ }^{31-33}$. Es importante señalar las diferencias con esos estudios que, a pesar de ser pediátricos, se realizaron en niños de mayor edad y distintos tamaños muestrales. Además, es conocida la subestimación o sobreestimación de la ingesta en niños con obesidad o desnutrición respectivamente, por parte de los padres o cuidadores.

En cuanto al consumo dietario fue significativamente mayor en aquellos que no incluían LM en su dieta y tenían entre 12 y 18 meses, esto es congruente con el tipo de registro realizado, ya que no se cuantificó el consumo de LM en nuestros niños, y solo se utilizó esta variable de manera cualitativa, mostrando en los niños entre 12 a 18 meses una proporción de niños con LM menor que en el grupo de 6 a 11 meses (38,7\% vs. $71 \%$, respectivamente). Los aportes de la dieta de los niños que consumían LM son similares a los observados en otro estudio, donde se encontró que la brecha de energía diaria a cubrir la alimentación complementaria en niños entre 6 a 8 meses es de 200 calorías, entre 9 a 11 meses de 300 calorías y entre 12 a 23 meses de 500 calorías, aproximadamente, sobre la base de un requerimiento energético aproximado de 600, 700 y 900 calorías respectivamente ${ }^{34}$.

$\mathrm{Al}$ comparar la ingesta versus los requerimientos de la muestra, se observó que el porcentaje de adecuación de energía fue normal según sexo, estado nutricional normal y MD, esto puede deberse a que la estimación de los requerimientos de energía calculados por ecuaciones teóricas tiende a la sobreestimación, lo que explicaría que las adecuaciones de energía en este estudio no se asocien con el estado nutricional ${ }^{21}$. Además, observamos que gran parte de los macronutrientes y micronutrientes no estuvieron adecuados en los rangos esperados. Cabe destacar lo observado con proteínas y carbohidratos, los cuales tuvieron una adecuación sobre lo esperado, siendo en las proteínas similar a lo observado en un estudio italiano en niños de 6, 9 y 12 meses $^{35}$, existen diversos estudios que asocian el exceso en la ingesta de estos macronutrientes y obesidad infantil. Esta relación se ha observado 
en lactantes que consumen fórmulas lácteas (FL) infantiles con alta concentración de proteínas ${ }^{36,37}$ y en conjunto con su alimentación complementaria ${ }^{38,39}$, donde se ha visto aumento del peso y grasa corporal. Los resultados concuerdan con un estudio nacional el cual mostró los problemas que existen en la dilución de las FL, siendo más habituales en el NSE bajo, en el que cerca de un $40 \%$ de los padres la concentraba por sobre lo recomendado en niños entre 6 a 11 meses $^{40}$. Respecto a los nutrientes que adecuaron por debajo de lo esperado, en los lípidos podría encontrarse un subregistro, además podría afectar el no registro cuantitativo de la LM que aporta en promedio $44 \%$ de sus calorías en forma de lípidos ${ }^{41}$; en cuanto al omega 3 se debe al déficit de consumo de productos marinos en la población chilena ${ }^{28}$; y por último, la vitamina $\mathrm{D}$ que presenta una alta biodisponibilidad en la leche materna y la exposición solar ${ }^{42}$, pero no es un nutriente altamente presente en alimentos de consumo habitual en niños pequeños, por este motivo debe ser agregada como fortificación en los alimentos o suplementada en presencia de déficit ${ }^{42}$. También, se puede observar una disminución significativa en la ingesta de potasio en los niños con estado nutricional de ME comparado con los normales, estudios muestran que una alimentación rica en potasio disminuye los valores de presión arterial, por lo tanto, se debe fomentar el consumo de frutas y verduras desde el inicio de la alimentación complementaria ${ }^{43}$.

Cabe resaltar que la adecuación de calcio, hierro y zinc en la muestra general, según sexo y estado nutricional, estuvieron dentro de rangos esperados, siendo micronutrientes críticos en esta etapa del crecimiento y desarrollo. Esto se puede relacionar con la inclusión habitual en la dieta de estos niños de lácteos, huevo, legumbres, proteínas de origen animal y cereales fortificados, lo mismo fue descrito en otros estudios en donde se evidenció una relación positiva en la ingesta de estos micronutrientes críticos en patrones alimentarios que predomina el consumo de fórmulas infantiles, lácteos y cereales fortificados, como también la diversidad en los alimentos predice una ingesta adecuada de estos micronutrientes críticos $^{4,45}$.

Los resultados de esta investigación respecto a la clasificación del estado nutricional muestran que la prevalencia de malnutrición por exceso fue menor, mientras que la de malnutrición por déficit fue mayor a las reportadas a nivel ministerial ${ }^{8}$, aun así, existe un alto porcentaje en esta muestra de niños con malnutrición por exceso, llegando a un 28,7\% entre los 6 a 18 meses. Estas diferencias, especialmente en sobrepeso y obesidad pueden estar dadas por el tipo de muestreo donde su incluyó un 16,6\% NSE ABC1, en quienes está descrita la menor prevalencia de malnutrición por exceso comparada con NSE más bajo ${ }^{23,46}$.
Dentro de las debilidades observadas destacamos que no se realizó un cálculo de tamaño de la muestra, pudiendo afectar los resultados de estado nutricional, por lo cual no es posible extrapolar los resultados a toda la población chilena. Además, se debe considerar el autorregistro digital por parte de los cuidadores de los recordatorios de 24 horas que puede verse influenciado por el desconocimiento de gramajes o porciones a pesar de la educación realizada, así como por la percepción de la ingesta en función al estado nutricional del niño que puede llevar a la sobreestimación o subestimación de la ingesta; la ausencia en la cuantificación de la leche materna; y finalmente el tiempo de aplicación del estudio, no puede considerar la ingesta a largo plazo. En esta misma línea creemos que evaluar el estado nutricional y principalmente la ingesta y comportamiento alimentario de niños entre 6 a 18 meses es una de las mayores fortalezas de este estudio, ya que permite tener una mirada inicial de las conductas nutricionales de esta población, en función de intervenir de manera oportuna en las características dietarias y su repercusión en problemas nutricionales en la infancia como en edades posteriores. Cabe destacar, que dentro de las fortalezas también se encuentra el detallado recordatorio de 24 horas que fue de 4 a 7 días y el tipo de software utilizado, lo que permitió la recolección y análisis de una gran cantidad de datos para la evaluación de la ingesta y comportamiento dietario durante la infancia.

\section{Conclusiones}

Este estudio de carácter descriptivo entrega información específica de la ingesta y adecuación energéti$\mathrm{ca}$, macronutrientes y micronutrientes, así como de los hábitos alimentarios y estado nutricional de la muestra evaluada. La educación alimentaria en etapas tempranas de la vida es crucial en el desarrollo del comportamiento alimentario, así como en la mantención de un adecuado estado nutricional. Por este motivo la caracterización real de la ingesta alimentaria de nuestros niños puede contribuir de manera eficiente a solucionar problemas de salud muy prevalentes en la población actual, como es la deficiencia de micronutrientes y la obesidad infantil y su rol como factor relevante en la presencia o desencadenante de enfermedades crónicas no transmisibles en la vida adulta.

\section{Responsabilidades Éticas}

Protección de personas y animales: Los autores declaran que los procedimientos seguidos se conformaron a las normas éticas del comité de experimentación hu- 
mana responsable y de acuerdo con la Asociación Médica Mundial y la Declaración de Helsinki.

Confidencialidad de los datos: Los autores declaran que han seguido los protocolos de su centro de trabajo sobre la publicación de datos de pacientes.

Derecho a la privacidad y consentimiento informado: Los autores han obtenido el consentimiento informado de los pacientes y/o sujetos referidos en el artículo. Este documento obra en poder del autor de correspondencia.

\section{Conflicto de intereses}

Los autores declaran no tener conflicto de intereses.

\section{Financiamiento}

Esta investigación fue financiada por Nestlé. La empresa no tuvo ninguna participación en el diseño, ejecución, análisis de los resultados ni publicación del estudio.

\section{Referencias}

1. Cribb VL, Warren JM, Emmett $\mathrm{PM}$. Contribution of inappropriate complementary foods to the salt intake of 8-month-old infants. Eur J Clin Nutr. 2012;66(1):104-10

2. Ahluwalia N, Herrick KA, Rossen LM, et al. Usual nutrient intakes of US infants and toddlers generally meet or exceed Dietary Reference Intakes: Findings from NHANES 2009-2012. Am J Clin Nutr. 2016;104(4):1167-74.

3. Agostoni C, Przyrembel H. The timing of introduction of complementary foods and later health. World Rev Nutr Diet. 2013;108:63-70.

4. World Health Organization. Estrategia mundial para la alimentación del lactante y del niño pequeño [Internet]. Organización Mundial de la Salud, editor. 2003. $30 \mathrm{p}$.

5. Minsal. Guía de alimentación del niño(a) menor de 2 años guía de alimentación hasta la adolescencia. Cuarta Edi. 2015.

6. World Health Organization. Essential Nutrition Actions: Improving maternal, newborn, infant and young child health and nutrition. 2013.

7. Fewtrell M, Bronsky J, Campoy C, et al. Complementary feeding: A position paper by the European Society for Paediatric Gastroenterology, Hepatology, and Nutrition (ESPGHAN) committee on nutrition. J Pediatr Gastroenterol Nutr. 2017;64:119-32.

8. Minsal. Vigilancia del estado nutricional de la población bajo control y de la lactancia materna en el Sistema Público de Salud de Chile. Diciembre 2016. 128 p.

9. Carletti C, Concina F, Pani P, et al. Agerelated trends in the diet of an infant's cohort in the northeast of Italy from six to twelve months of age. Nutrients. 2019;11(2).

10. Magnus P, Birke C, Vejrup K, et al. Cohort Profile Update: The Norwegian Mother and Child Cohort Study (MoBa). Int J Epidemiol. 2016;382-8.
11. Kroke A, Manz F, Kersting M, et al. The DONALD Study: History, current status and future perspectives. Eur J Nutr. 2004;43:45-54.

12. Golding G, Pembrey P, Jones J. ALSPAC - The Avon Longitudinal Study of Parents and Children I. Study methodology. Paediatr Perinat Epidemiol. 2001;15:7487.

13. Valent F, Mariuz M, Bin M, et al. Associations of Prenatal Mercury Exposure From Maternal Fish Consumption and Polyunsaturated Fatty Acids With Child Neurodevelopment: A Prospective Cohort Study in Italy. 2013;23:360-70.

14. Asociación Investigadores de Mercado. El Nivel Socio Económico Esomar: Manual de Aplicación. Santiago: Adimark; 2000.

15. Asociación Investigadores de Mercado. Descripción grupos socioeconómicos 2008. Santiago: Asociación chilena de empresas de investigación de mercado; 2008.

16. Castillo VO, Rozowski NJ, Cuevas MA, et al. Ingesta de nutrientes en adultos mayores de la comuna de Providencia, Santiago de Chile. Rev Med Chil. 2002;130:1335-42.

17. Minsal. Patrones de crecimiento para la evaluación nutricional de niños, niñas y adolescentes desde el nacimiento hasta los 19 años de edad. 2018. 92 p.

18. WHO Multicentre Growth Reference Study Group. WHO Child Growth Standards: Length/height-for-age, weightfor-age, weight-for-length, weight-forheight and body mass index-for-age: Methods and development. Geneva: World Health Organization. 2006. 312 p.

19. Hall KD, Ayuketah A, Brychta R, et al. Ultra-Processed Diets Cause Excess Calorie Intake and Weight Gain: An Inpatient Randomized Controlled Trial of Ad Libitum Food Intake. Cell Metab. 2019;30:67-77.e3

20. Fiolet T, Srour B, Sellem L, et al. Consumption of ultra-processed foods and cancer risk: Results from NutriNet-
Santé prospective cohort. BMJ. 2018;360.

21. CEPAL. Principios y aplicación de las nuevas necesidades de energía según el Comité de Expertos FAO/OMS 2004. 2007. 70 p.

22. Institute of Medicine. Dietary Reference Intakes: The Essential Guide to Nutrient Requirements. Washington, DC: The National Academies Press; 2006. 1344 p.

23. Pérez SV, Novalbos-Ruiz JP, RodríguezMartín A, et al. Implicaciones del nivel socioeconómico familiar sobre las conductas de riesgo en la obesidad isnfantojuvenil. Nutr Hosp. 2013;28:195160.

24. Villagrán Pérez S, Rodríguez-Martín A, Novalbos Ruiz JP, et al. Hábitos y estilos de vida modificables en niños con sobrepeso y obesidad. Nutr Hosp. 2010;25:823-31.

25. Fundación Chile. Chile saludable: oportunidades y desafíos de innovación para colaciones escolares saludables. Santiago - Chile; 2018. 113 p.

26. Martin SL, McCann JK, Gascoigne E, et al. Mixed-Methods Systematic Review of Behavioral Interventions in Low- And Middle-Income Countries to Increase Family Support for Maternal, Infant, and Young Child Nutrition During the First 1,000 Days. Curr Dev Nutr. 2020;1-27.

27. Crovetto M. Cambios en la estructura y consumo de alimentos en hogares del Gran Santiago 1988-1997. Rev Chil Nutr. 2002;29:24-32.

28. Minsal. Encuesta nacional de consumo alimentario. Informe Final. 2010. 329 p.

29. Minsal. Ley 20.606. Sobre composición nutricional de los alimentos y su publicidad. 2013

30. Quitral V, Arteaga J, Rivera M, et al. Comparación del contenido de azúcares y edulcorantes no calóricos en néctares y bebidas antes y después de implementar la ley chilena 20.606. Rev Chil Nutr. 2019;46:245-53.

31. Ledesma Ríos NI, Sepúlveda Herrera DM, Cárdenas Sánchez DL MCL. Ingesta 
de energía y nutrientes en niños de 2-4 años que asisten al programa "Buen Comienzo", Medellín (Colombia). Nutr Hosp. 2016;33:1052-61.

32. Telford RD, Cunningham RB, Telford RM, et al. Determinants of Childhood Adiposity: Evidence from the Australian LOOK Study. PLoS One. 2012;7:7-13.

33. Liberona ZY, Engler TV, Castillo $\mathrm{VO}$, et al. Ingesta de macronutrientes y prevalencia de malnutrición por exceso en escolares de $5^{\circ}$ y $6^{\circ}$ básico de distinto nivel socioeconómico de la región metropolitana. Rev Chil Nutr. 2008;35:190-9.

34. OPS. "La alimentación del lactante y del niño pequeño: Capítulo Modelo para libros de texto dirigidos a estudiantes de medicina y otras ciencias de la salud" [Internet]. Washington, D.C.: OPS; 2010.

35. Concina F, Pani P, Bravo G, et al. Nutrient intakes in an Italian population of infants during the complementary feeding period. Public Health Nutr. 2018;21:3018-26.

36. Patro-Gołąb B, Zalewski BM, Kouwenhoven SM, et al. Protein
Concentration in Milk Formula, Growth, and Later Risk of Obesity: A Systematic Review. J Nutr. 2016;146:551-64.

37. Fleddermann $\mathrm{M}$, Demmelmair $\mathrm{H}$, Hellmuth C, et al. Association of infant formula composition and anthropometry at 4 years: Follow-up of a randomized controlled trial (BeMIM study). PLoS One. 2018;13:1-14

38. Koletzko B, Von Kries R, Monasterolo $\mathrm{RC}$, et al. Can infant feeding choices modulate later obesity risk? Am J Clin Nutr. 2009;89:1502-9.

39. Brands B, Demmelmair H, Koletzko B. How growth due to infant nutrition influences obesity and later disease risk. Vol. 103, Acta Paediatrica, International Journal of Paediatrics. Blackwell Publishing Ltd; 2014;578-85.

40. Cornejo EV, Fernández GE, Castro ChG, et al. Study on dilution of three types of powderer milk in families belonging to socioeconomic groups: $\mathrm{ABC} 1, \mathrm{C} 2$ and $\mathrm{C} 3$ in Santiago. Rev Chil Nutr. 2007;34:35363.

41. Grote V, Verduci E, Scaglioni S, et al. Breast milk composition and infant nutrient intakes during the first
12 months of life. Eur J Clin Nutr. 2016;70:250-6.

42. Pani P, Carletti C, Knowles A, et al. Patterns of nutrients' intake at six months in the northeast of Italy: A cohort study. BMC Pediatr. 2014;14:127.

43. Saieh C, Lara M, Opazo M. Ingesta de sodio y potasio en niños de 0 a 15 años: lo observado versus lo esperado. Rev Médica Clínica Las Condes. 2015;26:537-43.

44. Faber M, Rothman M, Laubscher R. Dietary patterns of 6-24-month-old children are associated with nutrient content and quality of the diet. Matern Child Nutr. 2020;16:1-12.

45. Campbell RK, Hurley KM, Shamim AA, et al. Complementary food supplements increase dietary nutrient adequacy and do not replace home food consumption in children 6-18 months old in a randomized controlled trial in rural Bangladesh. J Nutr. 2018;148:1484-92.

46. Kain J, Orellana Y, Leyton B, et al. Association between Socioeconomic Vulnerability and Height with Obesity in Low-Income Chilean Children in the Transition from Preschool to First Grade. Ecol Food Nutr. 2014;53:241-55. 